\title{
Complications in the surgical management of femur fractures in children with non-ambulatory cerebral palsy
}

\author{
Dr M O'Brien MBBCh, FC (Orth)SA \\ Dr Y Ramguthy BMedSc, MBChB, FC (Orth)SA \\ Dr GB Firth MBBCh, FC (Orth)SA, MMed(Orth)(Wits)
}

Department of Orthopaedics, Chris Hani Baragwanath Academic Hospital, University of the Witwatersrand

\author{
Corresponding author: \\ Dr GB Firth \\ 7 York Road \\ Parktown \\ University of the Witwatersrand \\ Medical School \\ 2184 \\ Email: greg.firth@gmail.com
}

\begin{abstract}
Introduction
Children with severe non-ambulatory cerebral palsy (CP) are at high risk of sustaining a pathological femur fracture. ${ }^{1,2}$ These fractures are rare. In a review of the epidemiology of femur fractures in children from Cape Town, the prevalence of femoral pathological fractures related to cerebral palsy was less than three per cent. ${ }^{3}$ These patients often have multiple co-morbidities which predispose them to pathological femur fractures. ${ }^{3.6}$ The gold standard of treatment for these fractures is conservative management with either traction or cast immobilisation. The incidence of complications has been reported as extremely high in a number of studies after surgical management of these fractures. ${ }^{1,7}$ The objectives of this case report are to discuss the aetiology of these fractures, emphasise the role of conservative management as the gold standard of treatment, and highlight complications of surgical treatment in children with non-ambulatory cerebral palsy.
\end{abstract}

Key words: cerebral palsy, fracture, bone health

\section{Presentation}

ZS is a 16-year-old female with mixed CP, gross motor function classification system (GMFCS) level $\mathrm{V}$ and uncontrolled epilepsy. Her parents gave a history of bilateral clavicle fractures and a left femur fracture which was treated surgically with an open reduction and plate fixation in 2012. The current episode occurred after she fitted and fell from her bed. Although verbal communication was impossible it was clear that she was suffering from severe pain in the right thigh. Examination revealed a patient with severe total body $\mathrm{CP}$, poor nutritional status, multiple contractures involving the hip, knee and ankle with an extremely tender right groin and proximal thigh.
Plain X-rays of the pelvis and femurs showed the healed left midshaft femur fracture and the new right completely displaced sub-trochanteric femur fracture as shown in Figures 1 and 2.

The patient was treated surgically, with an open reduction and internal fixation using a blade plate for the right sub-trochanteric femur fracture and removal of the locked plate at the same surgical sitting.

The bone was extremely osteoporotic. She fitted again day 0 post-operatively, causing a re-fracture of her right femur resulting in the blade plate pulling out of her femoral neck (Figure 3). The left femur remained intact after removal of the locked plate at the same surgical sitting. 

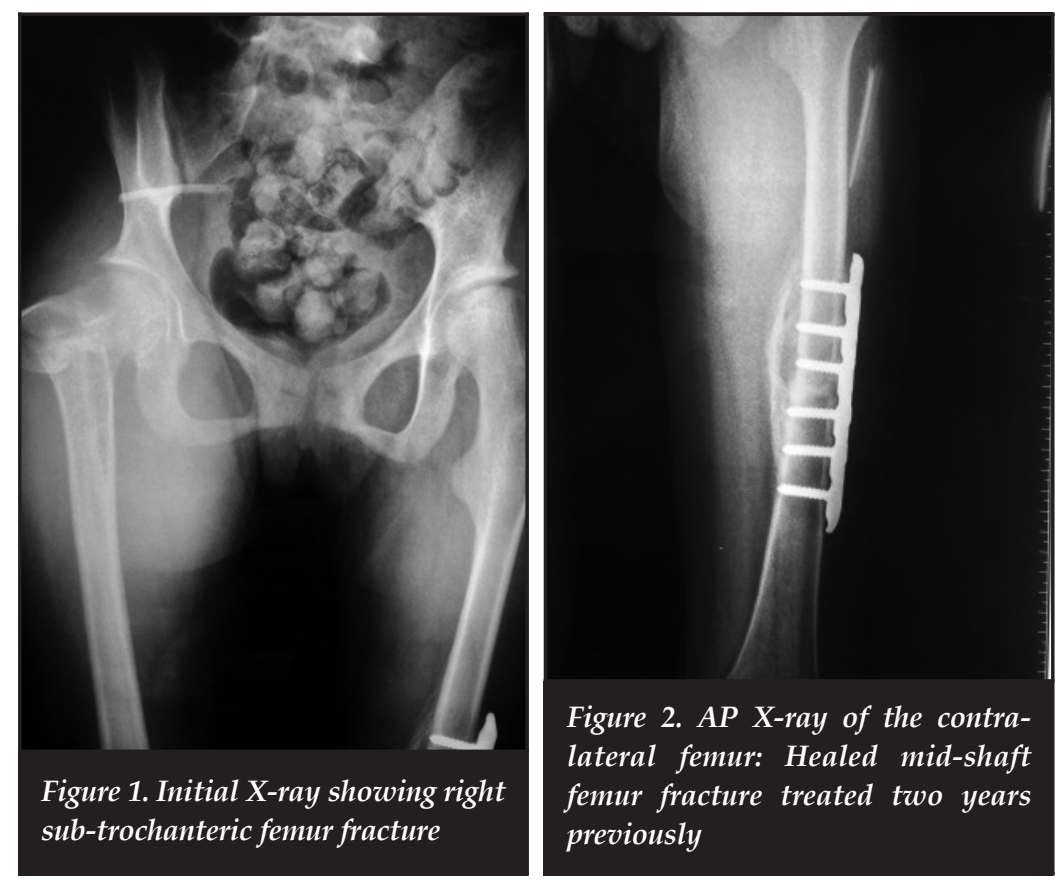

She continued to have breakthrough seizures, despite being reviewed by the paediatric neurology team, and being placed on therapeutic doses of an anticonvulsant, sodium valproate. She was subsequently taken back to theatre for a removal of hardware and a new internal fixation of the right sub-trochanteric femur fracture with a locked proximal femur plate (Figure 4). She remained stable in the ward despite occasional breakthrough seizures. Two weeks post revision open reduction and internal fixation with the locking plate she was discharged home in a stable condition.

Seizures can cause fractures in the setting of osteomalacia and osteoporotic bone, at the time of the seizure

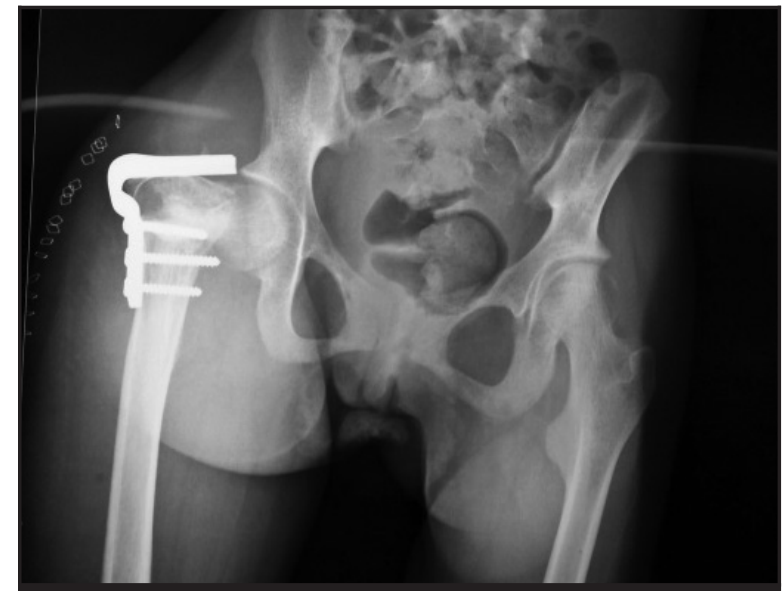

Figure 3. AP pelvis X-ray: After initial surgery the blade plate pulled out after a generalised tonic-clonic seizure.

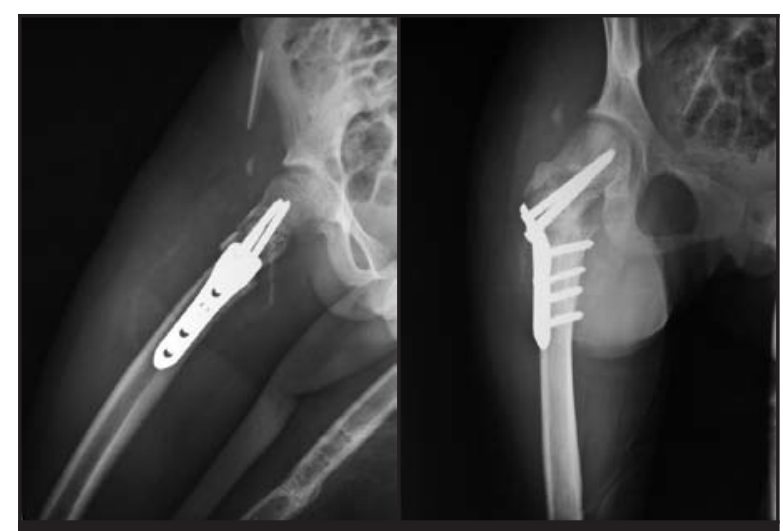

Figure 4. Post-operative revision surgery views of final surgical fixation using a locked proximal femoral plate
She returned to hospital two weeks after her discharge with a deep septic surgical wound site and a deteriorated general condition. The wound required four serial debridements and the use of a vacuum dressing in order to get the wound clean enough for secondary closure at the fifth surgery. She was given intravenous antibiotics including cloxacillin and tazobactam. Her in-patient management was complicated by septicaemia from Candida albicans which was cultured from the wound and blood culture. This was treated successfully with intravenous fluconazole, converted to oral medication on discharge. During her second admission a dual energy X-Ray absorptiometry (DEXA) scan was performed. This confirmed that she was severely osteoporotic, with a Z-score of -4.3 . After consultation with the paediatric metabolic unit, she was started on calcium and vitamin D. A decision was made not to start bisphosphonates. At six months follow-up her wounds had healed with no sign of infection and the fracture had united.

\section{Discussion}

Pathological fractures of the femur in adolescents with non-ambulatory $\mathrm{CP}$ are rare but well described. ${ }^{1-6}$ Uddenfeldt Wort et al. showed in a population study from 2013 that the risk of pathological fracture was significantly higher in children with non-ambulatory cerebral palsy, GMFCS IV or V compared with ambulatory children (GMFCS I-III). ${ }^{8}$

The aetiology of these fractures is multi-factorial. Vitamin D deficiency and overt rickets together with immobility, non-weight bearing and lack of exposure to sunlight have been closely associated. ${ }^{5,6,9}$ Replacement with vitamin $\mathrm{D}$ and calcium have been proposed by a number of studies to normalise levels. ${ }^{25,6}$ 
Epilepsy has also been shown to be a risk factor for pathological fractures. ${ }^{8}$ Seizures can cause fractures in the setting of osteomalacia and osteoporotic bone, at the time of the seizure, as in the current case. ${ }^{10}$ Anticonvulsant therapy (ACT) alters vitamin D metabolism by inducing the hepatic oxidase enzyme, thus further lowering vitamin D levels. ${ }^{2,5,11}$

Malnutrition due to a high catabolic rate and difficult feeding, together with central neurological causes, have also been cited as aetiological associations. ${ }^{12}$ Poor nutrition and stunted growth is well described in child ren with nonambulatory $\mathrm{CP}$.

Biomechanical abnormalities such as long lever arms and multiple joint contractures also play a contributory role in the aetiology of these fractures.

The assessment of low bone mineral density (BMD) in these patients is also associated with an increased risk of fragility fractures. ${ }^{13,14-16}$ Children with non-ambulatory $\mathrm{CP}$ should be assessed for osteoporosis. This can be done with the use of a DEXA scan using the distal femur as shown by Henderson et al. ${ }^{14,17}$ This method proved easier than the traditional hip and spine measurements and showed strong correlation with low bone mineral density (BMD) and pathological fractures in this patient population. Lower BMD Z scores, as in our patient, have been attributed to more severe GMFCS levels, difficult feeding and use of anticonvulsants. ${ }^{6}$ Sheridan found that in a large adult population of adults with disabilities the incidence of low bone density was 50 per cent. They also highlighted a better understanding required of the 'muscle-bone unit' physiology as a means to improving the outcome of fragility fractures in this population. ${ }^{18}$ Treatment with bisphosphonates is a relatively recent addition to the management of osteoporosis in these patients but has been shown to increase BMD and decrease fracture rates. ${ }^{9}$ There have, however, not been any studies comparing the effectiveness of bisphosphonates alone in comparison to sunlight, increased dietary calcium, vitamin D supplementation or adjustment of anti-convulsant therapy. ${ }^{9,19}$ Our patient did not receive bisphosphonates.

The patient in this case report had all of the above risk factors - contractures, immobility, resistant epilepsy, malnutrition, calcium and vitamin D deficiency and a low general physiological state which required close attention by a team of specialists. She was given calcium and vitamin D supplements but bisphosphonates were not given. Physiotherapists treated her while in hospital in an effort to mobilise her to the chair and to prevent pneumonia and pressure sores. A dietician was consulted to improve her poor nutritional status.
The patient in this case report had all of the risk factors contractures, immobility, resistant epilepsy, malnutrition, calcium and vitamin $D$ deficiency and a low general physiological state
The preferred orthopaedic treatment of femur fractures in children with non-ambulatory $\mathrm{CP}$ is conservative. ${ }^{1,3,7}$ Malunion in these patients is generally well tolerated as long as it does not interfere with perineal care and sitting balance. ${ }^{1,5}$ Conservative management in severe cerebral palsy has been described with good outcomes. McIvor et al. in 1966 reviewed 134 fractures in 92 patients with cerebral palsy. Over two-thirds of these fractures occurred in the femur and all were treated nonoperatively. Although $85 \%$ of these patients healed in a malunion, they found that none of the malunions interfered with the function of the patients. ${ }^{1}$ Leet et al. also showed that fractures in non-ambulatory patients with $\mathrm{CP}$ can be treated conservatively. ${ }^{7}$ Of 32 femur fractures treated conservatively, one-third healed in a malunion and five resulted in cast-related pressure sores. They emphasised that special attention should be paid to prevent high rates of significant malunion as shown in the article by McIvor et al. Significant malunion was defined as resulting in discomfort, seating or caring difficulties. The risk of non-union in this population was negligible in both studies. ${ }^{1,7}$ Conservative treatment should be regarded as the gold standard but careful attention must be paid to addressing causes for osteoporosis, controlling epilepsy and preventing complications, specifically pressure sores (either related to cast or bed immobility), malunion and pneumonia (either related to immobility or aspiration).

Surgical management in non-ambulatory children with $\mathrm{CP}$ has also been described. In a study by Leet et al., six femur fractures in non-ambulators were treated operatively. One resulted in a malunion, one developed a cast-related pressure sore, one developed pneumonia and one developed a bacteraemia giving an overall complication rate of $67 \% .^{7}$ Treating femur fractures in nonambulatory $\mathrm{CP}$ children with surgery is associated with a much higher complication rate and can be extremely challenging, as seen in our patient. Our initial primary goal with this patient was to alleviate her pain and allow her to be mobilised into a chair as soon as possible following the initial fracture. Surgical management in this case resulted in a high complication rate. Her seizure resulting in re-fracture requiring revision of her surgical fixation in extremely osteoporotic bone, and subsequent bacteraemia and deep wound sepsis highlights the major risk of complications in children with non-ambulatory cerebral palsy. Medical co-morbidities must be considered as well as low ambulatory potential, spasticity and osteopaenia. Leet et al. concluded that surgical management should be reserved for ambulatory $\mathrm{CP}$ children with unstable femur fractures. ${ }^{7}$

\section{Conclusion}

This case study highlights the extremely high complication rate with the surgical management of femoral fractures in children with non-ambulatory cerebral palsy. 
Although the conservative treatment of femoral fractures in these children is not without its complications, it should always be considered the gold standard. Epilepsy control, calcium and vitamin D supplementation have been shown to improve the bone health of these patients and should be considered as part of the management of femur fractures in children with non-ambulatory cerebral palsy.

The content of this article is the original work of the authors. No benefits of any form have been or are to be received from a commercial party related directly or indirectly to the subject of this article. Written permission was obtained from the patient and family to publish this case study.

\section{References}

1. McIvor WC, Samilson RL. Fractures in patients with cerebral palsy. J Bone Joint Surg (Am). 1966;48-A:858-66.

2. Lee JJK, Lyne ED. Pathological fractures in severely handicapped children and young adults. J Pediatr Orthop. 1990;10:497-500.

3. Mughal MA, Dix-Peek SI, Hoffman EB. The epidemiology of femur shaft fractures in children. SA Orthopaedic Journal. Summer 2013:23-27.

4. Brunner R, Doderlein L. Pathological fractures in patients with cerebral palsy. Journal of Pediatr Orthop B. 1996;5(4): 232-38.

5. Maree M, Dix-Peek S, Hoffman EB. The treatment and prevention of pathological fractures due to rickets in children with quadriplegic cerebral palsy and mental retardation. SA Orthopaedic Journal. May 2006:56-60.

6. Bischof F, Basu D, Pettifor JM. Pathological long-bone fractures in residents with cerebral palsy in a long-term care facility in South Africa. Dev Med Child Neurol. 2002;44(2):119-22.

7. Leet AI, Shirley ED, Barker C et al. Treatment of femur fractures in children with cerebral palsy. J Child Orthop. 2009; 3:253-58.

8. Uddenfeldt Wort U, Nordmark E, Wagner P et al. Fractures in children with cerebral palsy: a total population study. Dev Med Child Neurol. 2013 Sep;55(9):821-26.

9. Plotkin H, Coughlin S, Kreikemeier R et al. Low dose of pamidronate to treat osteopenia in children with severe cerebral palsy: a pilot study. Dev Med Child Neurol. 2006 Sep;48(9):709-12.
10. Vanheer $\mathrm{P}$, van Rhijn J, Boel M, et al. Supracondylar femur fracture complicating epileptic insult: a specific and under diagnosed complication?. Acta Neurol Belg. 2008 Mar;108(1):17-20.

11. Morijiri Y, Sato F. Factors causing rickets in institutionalised handicapped children on anticonvulsant therapy. Arch Dis Child 1981;56(6):446-49.

12. Ko $\mathrm{CH}$, Tse PW, Chan AK. Risk factors of long bone fractures in non-ambulatory cerebral palsy children. Hong Kong Med J. 2006 Dec;12(6):426-31.

13. Henderson RC, Lin PP, Greene WB. Bone mineral density in children who have spastic cerebral palsy. J Bone Joint Surg (Am). 1995;77-A:1671-79.

14. Henderson RC, Lark RK, Gurka MJ et al. Bone density and metabolism in children and adolescents with moderate to severe cerebral palsy. Pediatrics. 2002 Jul;110 (1 Pt1):e5.

15. Henderson RC, Kairalla JA, Abbas A, et al. Predicting low bone density in children and young adults with quadriplegic cerebral palsy. Dev Med Child Neurol. 2004;46:416-19.

16. Khoury DJ, Szalay EA. Bone mineral density correlation with fractures in nonambulatory pediatric patients. $J$ Pediatr Orthop. 2007;27:562-66.

17. Henderson RC, Berglund LM, May R et al. The relationship between fractures and DXA measures of BMD in the distal femur of children and adolescents with cerebral palsy or muscular dystrophy. J Bone Miner Res. 2010 Mar;25(3):520-26.

18. Sheridan KJ. Osteoporosis in adults with cerebral palsy. Dev Med Child Neurol. 2009 Oct;51 Suppl 4:38-51.

19. Sholas MG, Tann B, Gaebler-Spira D. Oral biphosphonates to treat disuse osteopenia in children with disabilities: A case series. J Pediatr Orthop. 2005;25:326-31.

This article is also available online on the SAOA website (www.saoa.org.za) and the SciELO website (www.scielo.org.za). Follow the directions on the Contents page of this journal to access it. 